\title{
Connecting Inflammation with Glutamate Agonism in Suicidality
}

Sophie Erhardt, Chai K. Lim, Klas R. Linderholm, Shorena Janelidze, Daniel Lindqvist, Martin Samuelsson, Kristina Lundberg, Teodor T Postolache, Lil Traskman-Bendz, Gilles J Guillemin and Lena Brundin

\section{Linköping University Post Print}

\section{Tweet}

N.B.: When citing this work, cite the original article.

Original Publication:

Sophie Erhardt, Chai K. Lim, Klas R. Linderholm, Shorena Janelidze, Daniel Lindqvist, Martin Samuelsson, Kristina Lundberg, Teodor T Postolache, Lil Traskman-Bendz, Gilles J Guillemin and Lena Brundin, Connecting Inflammation with Glutamate Agonism in Suicidality, 2013, Neuropsychopharmacology, (38), 5, 743-752.

http://dx.doi.org/10.1038/npp.2012.248

Copyright: Nature Publishing Group: Open Access Hybrid Model Option A http://www.nature.com/

Postprint available at: Linköping University Electronic Press http://urn.kb.se/resolve?urn=urn:nbn:se:liu:diva-91010 


\title{
Connecting Inflammation with Glutamate Agonism in Suicidality
}

\author{
Sophie Erhardt', Chai K Lim², Klas R Linderholm', Shorena Janelidze ${ }^{3}$, Daniel Lindqvist ${ }^{3}$, \\ Martin Samuelsson ${ }^{4}$, Kristina Lundberg ${ }^{4}$, Teodor T Postolache ${ }^{5}$, Lil Träskman-Bendz ${ }^{3}$, Gilles J Guillemin ${ }^{2,6,8}$ \\ and Lena Brundin*,3,7,8 \\ 'Department of Physiology and Pharmacology, Karolinska Institutet, Stockholm, Sweden; '2Department of Pharmacology, School of Medical \\ Sciences, University of New South Wales, NSW, Sydney, Australia; ${ }^{3}$ Department of Clinical Sciences, Section of Psychiatry, Psychoimmunology \\ Unit, Lund University, Lund, Sweden; ${ }^{4}$ Faculty of Health Sciences, Department of Clinical and Experimental Medicine, Division of Psychiatry, \\ Linköping University, Linköping, Sweden; ${ }^{5}$ Mood and Anxiety Program, University of Maryland School of Medicine and the VA Capitol Healthcare \\ Network (VISN 5) Mental Illness Research, Education and Clinical Center, Baltimore, MD, USA; ' ${ }^{6}$ ustralian School of Advanced Medicine \\ (ASAM), Macquarie University, NSW, Australia; ${ }^{7}$ Department of Translational Science and Molecular Medicine, Michigan State University, \\ Van Andel Research Institute, Grand Rapids, MI, USA
}

\begin{abstract}
The NMDA-receptor antagonist ketamine has proven efficient in reducing symptoms of suicidality, although the mechanisms explaining this effect have not been detailed in psychiatric patients. Recent evidence points towards a low-grade inflammation in brains of suicide victims. Inflammation leads to production of quinolinic acid (QUIN) and kynurenic acid (KYNA), an agonist and antagonist of the glutamatergic N-methyl-D-aspartate (NMDA) receptor, respectively. We here measured QUIN and KYNA in the cerebrospinal fluid (CSF) of 64 medication-free suicide attempters and 36 controls, using gas chromatography mass spectrometry and high-performance liquid chromatography. We assessed the patients clinically using the Suicide Intent Scale and the Montgomery-Asberg Depression Rating Scale (MADRS). We found that QUIN, but not KYNA, was significantly elevated in the CSF of suicide attempters $(P<0.00 \mathrm{I})$. As predicted, the increase in QUIN was associated with higher levels of CSF interleukin-6. Moreover, QUIN levels correlated with the total scores on Suicide Intent Scale. There was a significant decrease of QUIN in patients who came for follow-up lumbar punctures within 6 months after the suicide attempt. In summary, we here present clinical evidence of increased QUIN in the CSF of suicide attempters. An increased QUIN/KYNA quotient speaks in favor of an overall NMDA-receptor stimulation. The correlation between QUIN and the Suicide Intent Scale indicates that changes in glutamatergic neurotransmission could be specifically linked to suicidality. Our findings have important implications for the detection and specific treatment of suicidal patients, and might explain the observed remedial effects of ketamine.
\end{abstract}

Neuropsychopharmacology (2013) 38, 743-752; doi:I0.1038/npp.20I2.248; published online 9 January 2013

Keywords: quinolinic acid; suicide; depression; glutamate; cytokine

\section{INTRODUCTION}

Attempted suicide is associated with significant patient suffering, vast societal costs and an increased risk for completed suicide (Bruffaerts et al, 2011; Hawton and van Heeringen, 2009). Despite increased treatment of suicidal individuals over the past decade, the incidence rates of suicidal behavior have remained largely unaltered (Nock et al, 2008). Although many patients contact a physician shortly before the attempt, health care is frequently unable to prevent both attempted and completed suicides. Consequently, improved methods for detection of suicide risk and

*Correspondence: Dr L Brundin, Department of Translational Science and Molecular Medicine, Michigan State University, Van Andel Research Institute, 333 Bostwick Avenue NE, Grand Rapids, MI 49503, USA, Tel: + 616234 0996, Fax: + 616234 0990,

E-mail: Lena.Brundin@hc.msu.edu

${ }^{8}$ Shared senior authorship.

Received 22 August 2012; revised 2 November 2012; accepted 26 November 2012; accepted article preview online 3 December 2012 specific treatments for suicidal patients are both highly warranted (Da Cruz et al, 2011).

The neurobiology of suicidality is poorly understood. Biological factors that have previously been associated with suicidality include serotonin and its metabolite 5-hydroxyindoleacetic acid (5-HIAA) as well as growth factors, such as brain-derived neurotrophic factor (Sher, 2011). Certain studies have shown an association between low levels of CSF 5-HIAA and suicidal behavior independent of depression (Asberg and Traskman, 1981). Reduced serotonergic neurotransmission and suicidal behavior might thus be linked, but after more than 30 years of research, the association remains unspecific (Ernst et al, 2009).

Interestingly, production of serotonin might be compromised during states of inflammation due to a consumption of tryptophan, the substrate for serotonin production (Oxenkrug, 2010). Recent studies provide accumulating evidence that brain immune activation may be involved in the pathogenesis of suicidality. A post-mortem study shows brain microglia activation in suicide victims (Steiner et al, 
2008), and the cytokine IL- 6 is increased in the cerebrospinal fluid (CSF) of suicide attempters (Lindqvist et al, 2009). In addition, some studies indicate that inflammation is more pronounced in suicidal patients than in non-suicidal depressed patients (Janelidze et al, 2011; Steiner et al, 2008). In support of a causal relationship between inflammation and psychiatric symptoms, several studies show that immunotherapy with cytokines in patients induces depressive symptoms approximately 1 month after the beginning of the medication (Raison et al, 2009; Wichers et al, 2005). Several case reports also describe suicidal ideation in previously psychiatrically healthy individuals after treatment with interferon- $\beta$ (Fragoso et al, 2010).

Inflammatory stimuli, such as central nervous system (CNS) infections, induce the kynurenine pathway of tryptophan degradation (Figure 1) and greatly increase CSF levels of kynurenine and its metabolites (Heyes et al, 1992). Pro-inflammatory cytokines, especially interferon $\gamma$, are considered the major inducers of indoleamine-2,3dioxygenase (IDO-1), one of the enzymes regulating the first step of the kynurenine pathway (Guillemin, 2012). Metabolism further along this pathway produces several neuroactive compounds, including quinolinic acid (QUIN) and kynurenic acid (KYNA), both of which affect glutamatergic neurotransmission. A potential pathogenetic mechanism underlying suicidal behavior could thus be via activation of the kynurenine pathway and through increased synthesis of QUIN and/or KYNA. Blood kynurenine levels were recently found to be elevated in suicide attempters, as compared not only with healthy controls but also with patients with depression who never attempted suicide (Sublette et al, 2011).

QUIN is an $N$-methyl-D-aspartate (NMDA)-receptor agonist, activating receptors containing the NR1 + NR2A and the NR1 + NR2B subunits (de Carvalho et al, 1996; Stone, 1993). By contrast, KYNA, besides blocking the cholinergic $\alpha 7$ nicotinic receptor, antagonizes the glycine site of the NMDA-receptor (Hilmas et al, 2001; Stone, 1993). Interestingly, the NMDA-receptor antagonist ketamine was recently shown to reduce suicidality in four small-scale clinical studies (DiazGranados et al, 2010b; Larkin and Beautrais, 2011; Price et al, 2009; Zarate et al, 2012). This finding may principally suggest enhanced NMDA-receptor signaling as part of the pathophysiology of suicidal behavior, although ketamine also has other effects in the central nervous system (Duman and Aghajanian, 2012).

We hypothesize that QUIN, and the QUIN/KYNA quotient, is elevated in suicide attempters due to a lowgrade CNS inflammation. This might lead to excessive NMDA-receptor signaling, tentatively explaining the observed remedial effects of ketamine on suicidality. Here, CSF levels of QUIN and KYNA are measured in suicide attempters and healthy controls. Furthermore, we assess whether the QUIN levels are related to the depressive symptoms, and to suicidal intent using the Suicide Intent Scale (Beck, 1974). We also analyze whether QUIN and KYNA correlate with CSF levels of the cytokine IL-6, which we have previously reported elevated in the suicide attempters (Lindqvist et al, 2009). A subset of the patients returned to the clinic at a follow-up occasion within 6

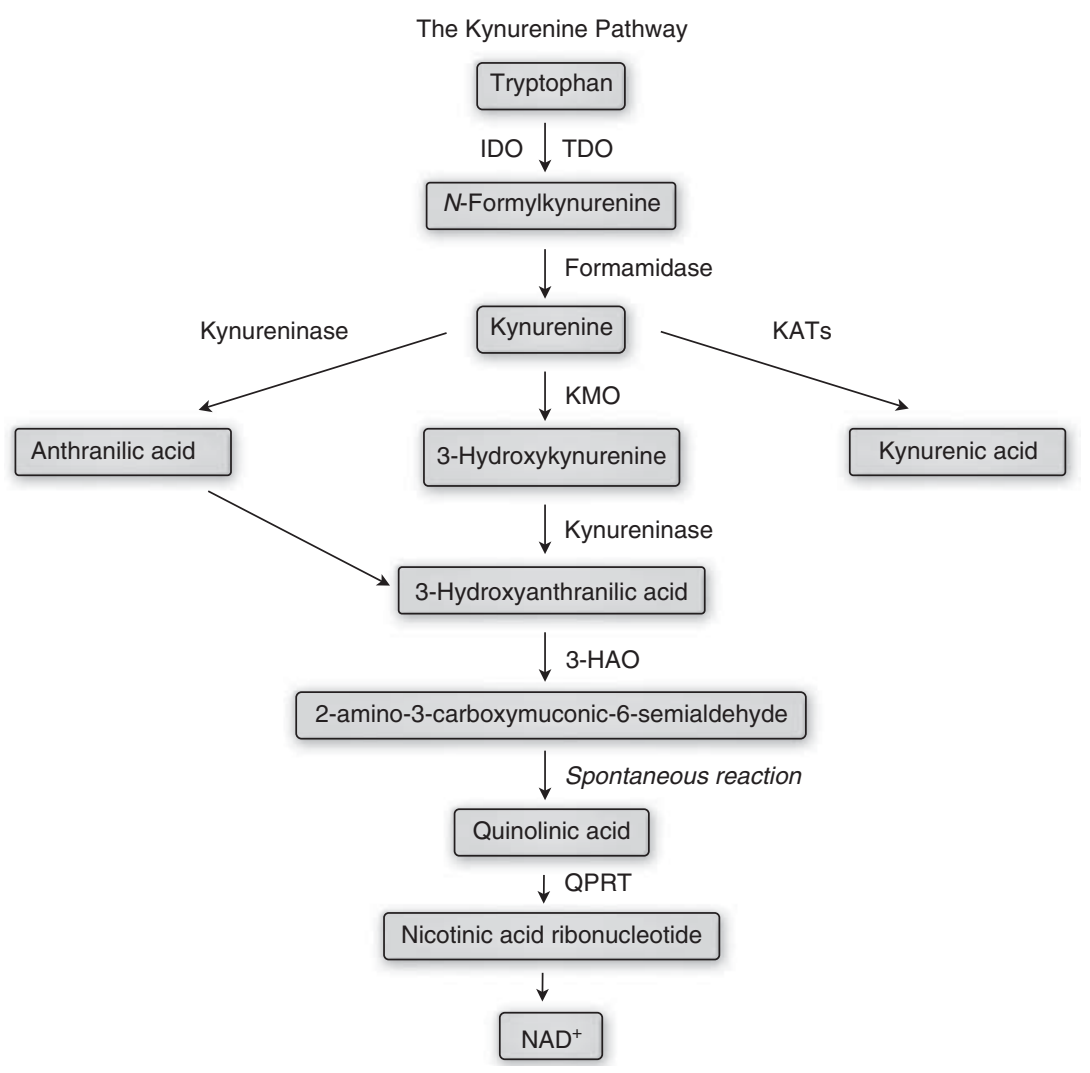

Figure I Simplified diagram of the kynurenine pathway. 
months of the suicide attempt and provided a new CSF sample. This enables us to determine whether QUIN and KYNA levels change longitudinally in the same patients, at a time-point when the patients were not suicidal.

\section{MATERIALS AND METHODS}

\section{Participants, QUIN CSF study}

We enrolled 64 patients (30 male and 34 female individuals), between 1988 and 2001, following admission to Lund University Hospital after a suicide attempt. Psychiatric diagnoses of the patients are displayed in Table 1. Mean age of the patients was 37 years (range 19-72). The patients underwent a washout period when they did not receive any antipsychotic or anti-depressive medication (14.6 \pm 9 days, mean \pm s.d.). Anxiolytic and hypnotic medications, as well as somatic medications, were allowed during the wash-out. All medications taken are specified in Supplementary Table 1 . At the end of the washout, lumbar punctures and psychiatric evaluations were carried out as below (section 2.2). Thirty-six (29 male and 7 female individuals) healthy controls were recruited via the Psychiatric Clinics at the University Hospitals in Lund and Linkoping, Sweden, between 2003 and 2009. Mean age of the controls was 30 years (range 18-66). They did not suffer from any previous or ongoing psychiatric condition or substance abuse, and were somatically healthy. They were thoroughly checked for psychiatric morbidity using the Structured Clinical Interviews for DSM Disorders (SCID I and II). All controls were free of medication. Our study was carried out in accordance with 'The code of ethics of the world medical association (Declaration of Helsinki)' for experiments including humans: http://www.wma.net/e/policy/b3.htm. The Regional Ethical Review Boards in Lund, Linköping and Malmö approved the study. After complete description of the study to the subjects, written informed consent was obtained.

\section{Physical examination and lumbar punctures}

All patients and controls underwent a general physical examination. The BMI for patients and controls were $23.7 \pm 3.5$ (mean \pm s.d.) and $24.2 \pm 3.9$, respectively. In all, $31 \%$ of the patients used nicotine $v s 19.4 \%$ of the controls. In order to identify subjects with potential infections at the time of the lumbar punctures, we analyzed blood samples for white blood cell count, erythrocyte sedimentation rate or
C-reactive protein, and the subjects were checked for fever. No evidence of ongoing clinical infection was found, as defined by the normal reference intervals of these parameters. A complete medical history was taken. Somatic diagnoses of the patients are shown in Table 1. We performed lumbar punctures in the morning between 0800 and 0900 hours, after a night of fasting and bed rest. CSF was collected from the L4-L5 interspace using a standardized protocol, and immediately stored at $-80^{\circ} \mathrm{C}$.

\section{Psychiatric diagnostics and rating scales}

Briefly after the suicide attempt, a psychiatrist diagnosed the patients according to the Diagnostic and Statistical Manual of Mental Disorders (DSM)-IIIR Axis I and II Disorders (American Psychiatric Association, 1987). The diagnoses were set after a $\sim 2$-hour long structured interview using the Comprehensive Psychiatric Rating Scale and the Structured Clinical Interview for DSM Disorders (SCID I and II). The patients were also evaluated by means of the Suicide Intent Scale, measuring the determination to commit suicide (Beck, 1974). The scale is subdivided in two parts, dealing with objective (active preparation) and subjective circumstances related to the attempt. Some, but not all studies also find an association between high scores on the scale and future completed suicide (Freedenthal, 2008; Stefansson et al, 2012). In all, 53 out of 64 patients completed the Suicide Intent Scale. Moreover, we evaluated depressive symptoms using the Montgomery-Asberg Depression Rating Scale (MADRS), which is a 10-item scale with a maximum score of 60 (Montgomery and Asberg, 1979). In all, 60 out of 64 patients completed the MADRS rating.

\section{Definition of suicide attempts}

A suicide attempt was defined as 'situations in which a person has performed an actually or seemingly lifethreatening behavior with the intent of jeopardizing his/ her life or to give the appearance of such intent, but which has not resulted in death' (Beck et al, 1973). The intent to commit suicide was explicit upon interview. Patients who did not state a clear intent were not enrolled. Suicide attempts were classified into violent and nonviolent acts as defined (Traskman et al, 1981). Drug overdoses by ingestion, single wrist-cuts or a combination are considered nonviolent suicide attempts, whereas all other methods (for example, hanging, drowning, gas poisoning, several deep cuts) are classified as violent.

Table I Psychiatric and Somatic Disorders Among the Patients $(n=64)$

\begin{tabular}{lccl}
\hline Main axis I psychiatric diagnosis & Total number & Axis II, $\boldsymbol{n}(\%)$ & Somatic disorders \\
\hline Major depression & 22 & $10(46)$ & Migraine $(n=1)$, gastritis $(n=1)$ \\
Dysthymia & 4 & $4(100)$ & Gastritis $(n=1)$ \\
Depression not otherwise specified & 9 & $6(67)$ & Migraine $(n=1)$, gastritis $(n=1)$ \\
Adjustment disorders & $1 \mathrm{I}$ & $5(46)$ & Diabetes type II $(n=1)$, tension headache $(n=1)$ \\
Substance abuse disorder & 6 & $4(67)$ & \\
Psychosis & 3 & I (33) & \\
Anxiety disorders & 2 & $7(50)$ & \\
No axis I diagnosis & 7 & $100)$ & \\
\hline
\end{tabular}




\section{Participants, follow-up study}

A subset of the patients that were originally enrolled in the study at the suicide attempt participated in a follow-up study. The patients returned to the clinic and contributed with CSF samples at repeated occasions after the suicide attempt. Paired samples from eight patients, three male and five female individuals, were available from the index (the suicide attempts) and a time-point within 6 months after the attempt. Mean age of these patients was 38 years, range 22-51 years. Five of the patients received antidepressant medication at the follow-up occasion and one patient received disulfiram.

\section{Biological assays}

Analysis of QUIN. QUIN was analyzed by gas chromatography mass spectrometry as previously described (Smythe et al, 2002). The internal standard, $\left[{ }^{2} \mathrm{H}_{3}\right]$ QUIN $(99 \%)$ was purchased from Le Research Inc. (St Paul, MN, USA). Trifluoroacetic anhydride and 1,1,1,3,3,3-hexafluoro-2-propanol of GC derivatization grade, QUIN and other organic solvents of analytical-grade were all obtained from SigmaAldrich (St Louis, MO, USA). We injected $1 \mu$ of sample into an Agilent 6890 gas chromatograph, interfaced to an Agilent 5973 mass selective detector via an auto-sampler Agilent Technologies 7683 operating in negative ionization mode, and controlled using Agilent ChemStation software (Agilent, Santa Clara, CA, USA). Inter- and intra-assay precision is consistently $5-8 \%$.

Analysis of KYNA. KYNA was analyzed as previously described (Olsson et al, 2010) utilizing an isocratic reversed-phase high-performance liquid chromatography system, including a dual piston, high liquid delivery pump (Bischoff, Leonberg, Germany), a ReproSil-Pur C18 column (silica pore size, $3 \mu \mathrm{m}(4 \times 100 \mathrm{~mm}$, Dr Maisch $\mathrm{GmbH}$, Ammerbuch, Germany) and a fluorescence detector (Jasco Ltd, Hachioji City, Japan) with an excitation wavelength of $344 \mathrm{~nm}$ and an emission wavelength of $398 \mathrm{~nm}$ (18 nm bandwidth). A mobile phase of $50 \mathrm{~mm}$ sodium acetate ( $\mathrm{pH}$ 6.2 , adjusted with acetic acid) and $7.0 \%$ acetonitrile was pumped through the reversed-phase column at a flow rate of $0.5 \mathrm{ml} / \mathrm{min} .50 \mu \mathrm{l} \mathrm{samples} \mathrm{were} \mathrm{manually} \mathrm{injected} \mathrm{(ECOM,}$ Prague, Czech Republic). $0.5 \mathrm{M}$ zinc acetate (not $\mathrm{pH}$ adjusted) was delivered post-column by a peristaltic pump (P-500, Pharmacia, Uppsala, Sweden) at a flow rate of $0.10 \mathrm{ml} / \mathrm{min}$. The signals from the fluorescence detector were transferred to a computer for analysis with Datalys Azur (Grenoble, France). Retention time of KYNA was 7-8 min. Standard concentrations were used to relate the height of the peaks in the chromatogram to the correct concentration of KYNA in the samples. Inter- and intraassay precision is consistently $3-8 \%$. For KYNA analysis, CSF was available from 60 patients and 37 controls.

Analysis of IL-6. We analyzed IL-6 in CSF using highsensitivity electrochemiluminescence (MesoScale Discovery, Gaithersburg, Maryland, USA) as per the manufacturer's protocol. CSF samples were analyzed in duplicates on a SECTOR 6000 instrument (www.mesoscale.com). The detection limit was $0.1 \mathrm{pg} / \mathrm{ml}$. The absolute IL-6 measures have been published elsewhere (Lindqvist et al, 2009). Here, the correlation with QUIN was analyzed. For the correlation analyses, IL- 6 measures from 63 patients and 24 controls were available.

Statistical analysis and potential confounders. The Statistical Package for the Social Sciences (SPSS) program version 18.00 for Windows was used (IBM Corporation, New York, NY, USA). CSF levels of IL-6 and QUIN displayed skewness $>2$, and the values were transformed into normal distribution using the natural logarithms for statistical analysis. The potential impacts of age, gender, weight, BMI, smoking and length of wash-out period on QUIN and KYNA levels were investigated with Student's $t$-tests and Pearson correlations. To correct for age, linear regression models were used with QUIN as dependent variable and age as independent variable. Student's $t$-tests were used for comparisons of KYNA and age-corrected QUIN between independent groups. As KYNA and QUIN have opposing effects on the NMDA receptor, the ratio between CSF QUIN (age-corrected) and CSF KYNA (CSF QUIN/CSF KYNA) was calculated and the ratio was compared between suicide attempters and controls with Student's $t$-tests. In addition, linear regression analyses were conducted to determine the effect of sample storage time: QUIN or KYNA were entered as dependent variable, whereas group (patients $v s$ controls), age and sample storage time as independent variables. Age-corrected QUIN was compared between healthy controls, suicide attempters with a diagnosis of a primary mood disorder (major depressive disorder and depression NOS) and suicide attempters with other diagnoses using one-way ANOVA followed by Bonferroni-Dunn's post-hoc test. Age-corrected QUIN was also compared between the control group and five main diagnostic groups (Major Depressive Disorder, Dysthymia, Adjustment Disorder, Substance Abuse, Depression NOS) using one-way ANOVA. Finally, the impact of personality disorder, type of suicide attempt and wash-out on age-corrected QUIN were determined using one-way ANOVAs. Spearman's $\rho$ was used for correlation analysis of age-corrected QUIN and scores on the Suicide Intent Scale, as well as MADRS. Paired $t$-tests were used for QUIN, KYNA and MADRS measures within the same individuals in the follow-up study. $\alpha$-level of significance was set at $P=0.05$.

\section{RESULTS}

\section{CSF Levels of QUIN and KYNA}

We found that CSF QUIN was significantly higher in suicide attempters $(41.9 \pm 4 \mathrm{nM}$; mean \pm s.e.m.; $n=64)$ than in healthy controls $(18.2 \pm 0.8 \mathrm{nM} ; n=36)$ (Student's $t$-test, $t=-5.39, \mathrm{df}=93.88, P<0.001$ ) (Figure 2a). In contrast, there was no significant difference in the levels of CSF KYNA between suicide attempters and healthy controls (Student's $t$-test, $t=0.429, \mathrm{df}=93.70, P=0.67$ ) (Figure $2 \mathrm{~b}$ ). The concentrations of the respective metabolites are shown in Table 2.

As KYNA and QUIN have opposing effects on the NMDA receptor, we investigated the CSF QUIN/CSF KYNA ratio. We found that this ratio was significantly larger in the 

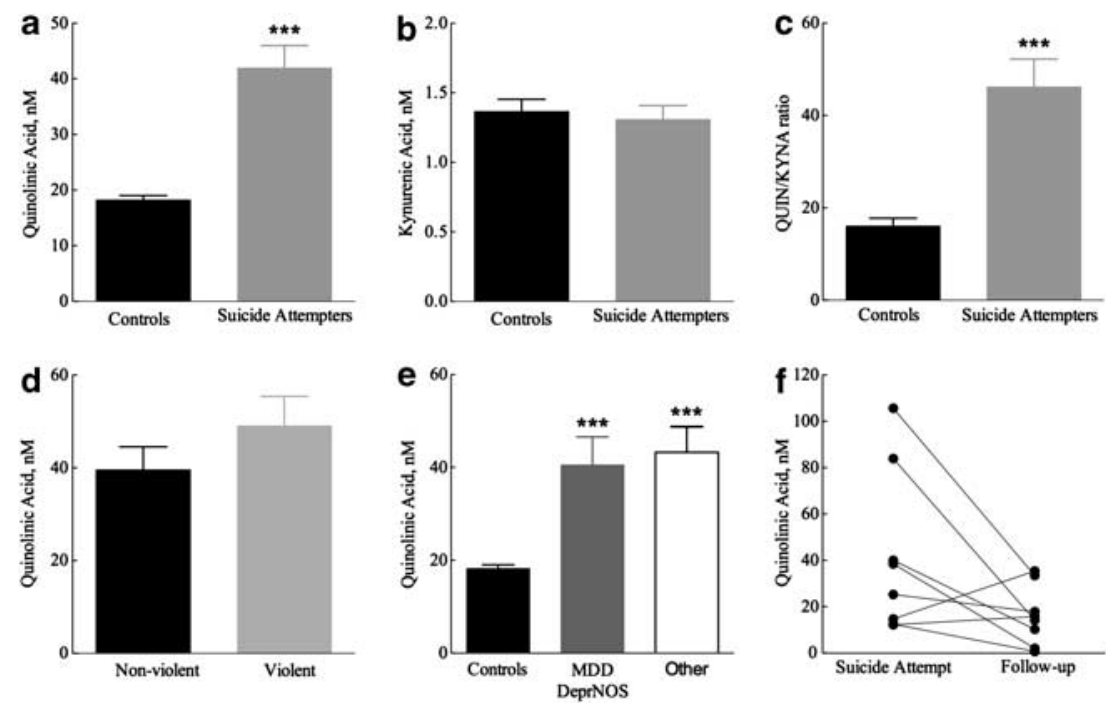

Figure 2 Quinolinic acid and kynurenic acid in cerebrospinal fluid of suicide attempters and healthy controls. (a) Cerebrospinal fluid quinolinic acid. (b) Cerebrospinal fluid kynurenic acid. (c) The quinolinic acid/kynurenic acid quotient. (d) Cerebrospinal fluid quinolinic acid in violent and nonviolent suicide attempters. (e) Cerebrospinal fluid quinolinic acid in suicide attempters with primary mood disorder (Major Depressive Disorder and Depression NOS), with other diagnoses and in controls. (f) Cerebrospinal fluid quinolinic acid in suicide attempters in the follow-up study. The lines connect measures of cerebrospinal fluid quinolinic acid from the same patients, between the suicide attempt and at 6 months after the attempt. The mean values + s.e.m. are shown for patients and controls, respectively, in figures (a-e) * $P \leqslant 0.05 ; * * P \leqslant 0.01$; **** $P \leqslant 0.001$

Table 2 Absolute CSF Concentrations of the Quinolinic (QUIN) Acid and Kynurenic Acid (KYNA) in Patients and Controls. Data are Presented as Mean \pm SEM

\begin{tabular}{lcccc}
\hline & QUIN, $\boldsymbol{n M}$ & $\boldsymbol{P}^{\mathbf{a}}$ & KYNA, $\boldsymbol{n M}$ & $\boldsymbol{p}^{\mathbf{a}}$ \\
\hline Controls & $18.22 \pm 0.79$ & $<0.001$ & $1.37 \pm 0.09$ & NS \\
Patients & $41.94 \pm 4.05$ & & $1.31 \pm 0.10$ & \\
MDD and deprNOS & $40.54 \pm 6.03$ & 0.001 & $1.25 \pm 0.14$ & NS \\
Other diagnosis & $43.26 \pm 5.51$ & $<0.001$ & $1.37 \pm 0.14$ & NS \\
Violent & $49.02 \pm 6.39$ & $<0.001$ & $1.20 \pm 0.17$ & NS \\
Nonviolent & $39.58 \pm 4.95$ & 0.002 & $1.34 \pm 0.12$ & NS \\
Unmedicated & $46.98 \pm 5.26$ & 0.003 & $1.30 \pm 0.15$ & NS \\
Medicated & $41.42 \pm 4.44$ & $<0.001$ & $1.31 \pm 0.11$ & NS \\
Personality disorder & $40.63 \pm 4.56$ & $<0.001$ & $1.34 \pm 0.15$ & NS \\
No personality disorder & $48.12 \pm 8.54$ & $<0.001$ & $1.22 \pm 0.14$ & NS \\
\hline
\end{tabular}

${ }^{a}$ Statistical analysis was done on age-corrected InQUIN data. All P-values represent comparisons vs the control group, after Bonferroni-Dunn correction when applicable.

suicidal patients than in the healthy controls (Student's $t$-test, $t=-3.72, \mathrm{df}=74.41, P<0.001$ ) (Figure $2 \mathrm{c}$ ). There was also a significant positive correlation between the cytokine IL-6 in CSF and QUIN ( $n=87$, Pearson's $R=0.23$, $P=0.033$ ) (Figure 3a).

\section{Demographics and potential confounders}

We investigated the potential impact of age, gender, weight, BMI, smoking, storage-time and length of wash-out period on QUIN and KYNA levels. QUIN showed a slight but significant increase with age of the subjects $(n=100$,
Pearson's $R=0.21, P=0.038$ ) (Figure $3 \mathrm{~b}$ ). We therefore used age-corrected variables for all statistical analyses except the within-subject analysis in the follow-up study (section 3.4). Age of the subjects did not correlate with KYNA ( $n=97$, Pearson's $R=0.043, P=0.68$ ) (Figure $3 \mathrm{c}$ ). There was no impact of gender, smoking, weight, BMI or wash-out length on QUIN nor KYNA ( $t$-tests and Pearson correlations, $P>0.1$ for all variables).

As the patient samples had been stored for a long time in our biobank, we also conducted a linear regression analyses to see if storage time had affected the levels of QUIN and KYNA. The model was still highly significant; $\beta=0.81$, $P=0.001$ for suicidality (patients $v s$ controls). There was a trend for a storage dependent decline of CSF QUIN; $\beta=-0.41, P=0.075$ for storage-time (Figure $3 \mathrm{~d}$ ). Thus, the QUIN levels in the samples from the suicide attempters in the biobank were potentially even higher initially. There was no effect of storage-time on CSF-KYNA (linear regression, $P>0.1$ ).

Both patients who were unmedicated at the suicide attempt $(n=6)$ and those who went through the wash-out $(n=58)$ had significantly elevated QUIN levels compared with the controls (one-way ANOVA $(\mathrm{F}(2,97)=11.15$, $P<0.001$; followed by Bonferroni-Dunn's post-hoc tests, $P=0.003$ for unmedicated $v s$ controls; $P<0.001$ for washedout $v s$ controls; NS for washed-out $v s$ unmedicated) (Table 2).

\section{QUIN, suicidality and depressive symptoms}

The mean score on the SIS was $18.3 \pm 0.8$ ( \pm s.e.m.). We found that QUIN measures in CSF correlated positively with the total score on the Suicide Intent Scale $(n=53$, Spearman $\rho=0.30, P=0.028$ ) (Figure 3e). The most robust correlation was between QUIN and the subscale concerning objective circumstances in conjunction with the suicide attempt 

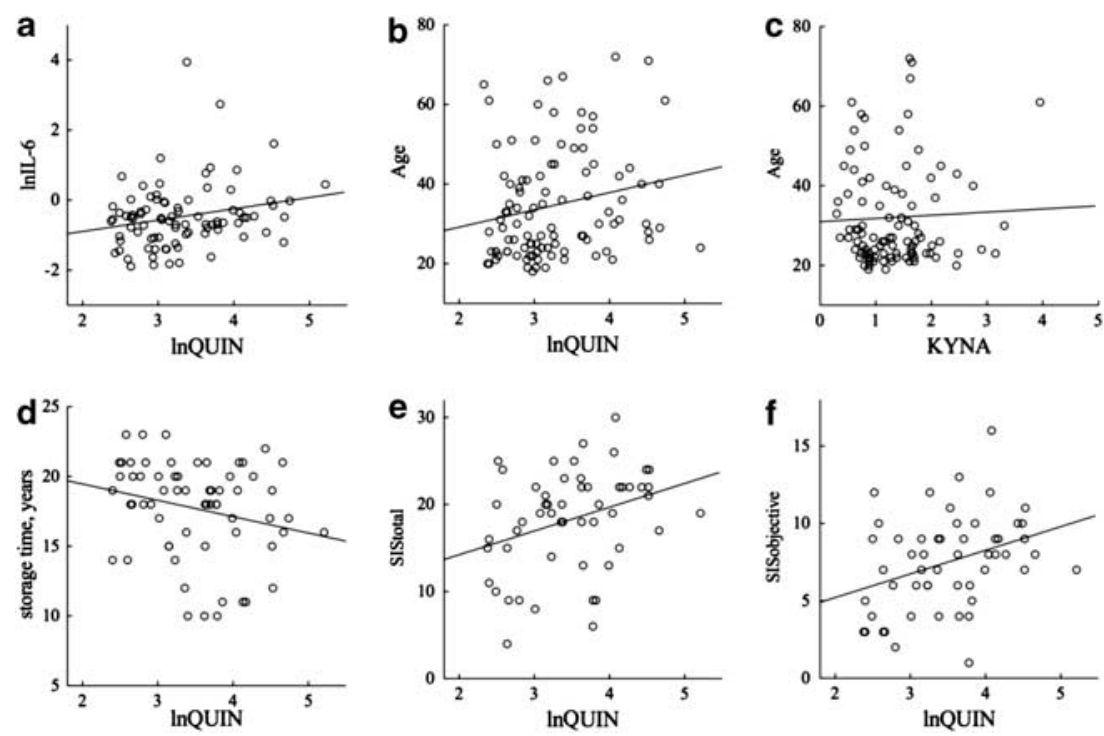

Figure 3 Associations of quinolinic acid with IL-6, age, sample storage time and suicidality; and kynurenic acid with age. Associations between In-quinolinic acid and (a) In-IL-6 ( $n=87$, Pearson's $R=0.23, P=0.033$ ), data from patients and controls; (b) age $(n=100$, Pearson's $R=0.21, P<0.0038)$ data from patients and controls; (d) sample storage time (linear regression model, $\beta=-0.4 \mathrm{I}, P=0.075$ ); (e) total scores on the Suicide Intent Scale (SIS) $(n=53$, Spearman $\rho=0.30, P=0.028)$, data from patients only; and ( $f$ ) scores on the subscale on the SIS rating objective circumstances of the suicide attempt $(n=53$, Spearman $\rho=0.34, P=0.012)$, data from patients only. (c) Association between kynurenic acid and age ( $n=97$, Pearson's $R=0.043, P=0.68)$.

( $n=53$, Spearman $\rho=0.34, P=0.012$ ) (Figure 3f). The definition of the objective circumstances can be found in section 2.3 .

CSF samples from suicide attempters were furthermore divided with regards to the use of violent $(n=16)$ or nonviolent $(n=48)$ methods for the attempts, as defined in the 'Materials and Methods' section. QUIN levels differed significantly between control subject, violent suicide attempters and nonviolent suicide attempters (one-way ANOVA $(\mathrm{F}(2,97)=13.16, P<0.001)$. There was a trend for higher levels of CSF QUIN in patients who had made violent suicide attempts than the patients who had made nonviolent suicide attempts (Bonferroni-Dunn's post-hoc test, $P=0.059$ ) (Figure $2 \mathrm{~d}$ and Table 2). Both violent and nonviolent attempter groups showed higher QUIN compared with the control group (Bonferroni-Dunn's post-hoc test, $P<0.001$ for violent attempters $v s$ controls and $P=0.002$ for nonviolent attempters $v s$ controls).

The mean score on the MADRS was $16.3 \pm 1.4$ ( \pm s.e.m.). We found no significant correlation between QUIN and the MADRS scores $(n=60$, Spearman $\rho=0.006, P=0.97)$. There was no correlation between QUIN and MADRS suicidality item ( $n=60$, Spearman $\rho=0.072, P=0.59)$.

\section{QUIN in diagnostic subgroups}

We compared the QUIN levels in suicide attempters with a diagnosis of a primary mood disorder (Major Depressive Disorder and Depression NOS $(n=31)$ with suicide attempters with other diagnoses $(n=33)$ and controls. A one-way ANOVA showed significant differences between the groups $(F(2,97)=9.81, P<0.001)$. Post-hoc testing showed that there was no significant difference in mean QUIN levels between suicide attempters with and without a mood disorder (NS), whereas both groups differed significantly $v s$ the healthy controls $(P=0.001$ for primary mood disorder vs controls; $P<0.001$ other diagnoses $v s$ controls; Bonferroni-Dunn's post-hoc tests) (Figure 2e and Table 2).

We also found significant differences in QUIN levels when comparing control group and the five main diagnostic groups of suicide attempters including Major Depressive Disorder, $n=22$; Adjustment Disorder, $n=11$; Depression NOS, $n=9$; Substance Abuse, $n=6$ and Dysthymia, $n=4$ (one-way ANOVA, $(\mathrm{F}(5,82)=4.64, P=0.001)$. Post-hoc testing showed that QUIN levels were not different between the five main diagnostic groups (Bonferroni-Dunn's posthoc test, $p>0.1$ for all comparisons).

QUIN levels did not differ between suicide attempters with $(n=38)$ and without $(n=22)$ personality disorder, but was higher in both groups compared with controls (oneway ANOVA $(\mathrm{F}(2,93)=11.42, \quad P<0.001$; followed by Bonferroni-Dunn's post-hoc test, $P>0.1$ for personality disorder $v s$ no personality disorder; $P<0.001$ for personality disorder $v s$ controls and $P<0.001$ for no personality disorder $v s$ controls) (Table 2).

\section{QUIN levels 6 months after the suicide attempt (follow-up)}

The CSF QUIN levels decreased significantly from the time of the suicide attempt to the follow-up occasion, from $41.6 \pm 12.4 \mathrm{~nm}$ to $16.2 \pm 4.5 \mathrm{~nm}$ (mean \pm s.e.m.) (paired samples $t$-test, $t=2.39, \mathrm{df}=7, P<0.05$ ) (Figure $2 \mathrm{f}$ ). Thus, CSF QUIN in the patients at follow-up had normalized to that of controls. There was no significant change in CSF KYNA, which decreased from $1.3 \pm 0.3 \mathrm{~nm}$ to $1.2 \pm 0.3 \mathrm{~nm}$ (mean \pm s.e.m.) (paired samples $t$-test, $t=0.37, \mathrm{df}=7, P=0.72$ ). There was a trend for a decrease in MADRS scores, from $16.8 \pm 2.0$ (mean \pm s.e.m.) at the suicide attempt to $10.0 \pm 4.3$ 
(mean \pm s.e.m.) at the follow-up occasion, in these eight patients (paired samples $t$-test, $t=2.0, \mathrm{df}=7, P=0.09$ ).

\section{DISCUSSION}

We found significantly increased levels of the NMDAreceptor agonist QUIN in the CSF of suicide attempters. In line with previous studies showing that inflammation can trigger generation of QUIN (Achim et al, 1993; Heyes et al, 1995), there was a significant correlation between CSF levels of QUIN and the pro-inflammatory cytokine IL-6. In contrast, levels of the NMDA-receptor antagonist KYNA were not elevated. This suggests that an overall agonistic effect on the NMDA-receptors is present in suicide attempters. Moreover, this finding provides a neurobiological rationale for the recent reports describing that ketamine, by blocking NMDA-receptor transmission, can alleviate symptoms of suicidality (DiazGranados et al, 2010b; Larkin and Beautrais, 2011; Price et al, 2009; Zarate et al, 2012). In our patients, the raised QUIN levels were also significantly associated with the degree of suicidal intent, further strengthening the association between QUIN and suicidality.

KYNA, which is mainly synthesized in astrocytes, blocks NMDA receptors and has neuroprotective and anticonvulsive properties (Erhardt et al, 2009; Schwarcz et al, 2012). In contrast, QUIN, which is produced by activated microglia, is a potent excitotoxin with neurotoxic, gliotoxic and proinflammatory properties (Guillemin, 2012; Schwarcz et al, 2012). Increased levels of QUIN might therefore potentially contribute to the neuronal loss and to the reduced hippocampal volume observed in patients with major depression (Bremner et al, 2000; McKinnon et al, 2009). Moreover, increased extracellular levels of QUIN, as reflected by increased CSF concentrations in suicide attempters, are likely to facilitate glutamate neurotransmission prior to causing apoptosis. In addition to being a direct NMDA receptor agonist, QUIN increases neuronal glutamate release as well as decreases glutamate uptake and recycling by astrocytes (Guillemin, 2012). Effects on the glutamate system have been suggested to contribute to the pathogenesis in patients with severe mood disorders (Hashimoto, 2009; Sanacora et al, 2008; Schwarcz et al, 2012). There has been no evidence of increased CSF QUIN in patients with primary depressive disorders or suicidality until now. In line with our findings, Steiner et al, (2011) recently observed increased QUIN-immunoreactivity in microglia in the anterior cingulate gyrus post-mortem of suicide victims that suffered from severe depression. Interestingly, the increase in CSF QUIN observed in our current study was not related to the severity of depressive symptoms. In agreement with this, QUIN levels were increased in all suicide attempters, regardless if they had a diagnosis of a mood disorder or not. Therefore, our study may suggest that elevated QUIN is specifically linked to suicidality rather than to the severity of depression. However, no non-suicide attempters with depressive symptoms were included in our study. Therefore, inflammation and subsequent stimulation of glutamate neurotransmission could potentially be prevalent in so-called treatment-resistant or severe depression; diagnostic groups where suicides, attempts and suicidal ideation all are common (Brent et al, 2009; Price et al, 2009; Steiner et al, 2011). There are several studies showing an effect of ketamine on patients with treatment-resistant depression, where suicidality has not specifically been an outcome measure (Diazgranados et al, 2010a; Ibrahim et al, 2012; Murrough et al, 2012). Further studies evaluating CSF QUIN in diagnostic subgroups should be undertaken in order to develop tailor-made treatments in the future.

It should be pointed out that we did not study any causal relation between biological factors and symptoms. This study analyzes associations and significant changes in primary psychiatric patients and controls. Important studies showing a causal relation between inflammatory factors and depressive symptoms have previously been performed in patients with hepatitis $\mathrm{C}$, and demonstrated that peripheral injection with interferon- $\alpha$ increased CSF levels of QUIN, KYNA and pro-inflammatory cytokines along with depressive symptoms (Raison et al, 2010; Raison et al, 2009).

In addition to the elevation in QUIN at the suicide attempt, we here also found a decrease of CSF QUIN in the eight patients who returned for a follow-up lumbar puncture within 6 months of the attempt. At this time-point, there was also a trend towards decreased depressive symptoms. However, the follow-up study sample was small, and the patients had initiated different types of medications. It is thus not possible to draw any conclusions about what caused the decrease in QUIN at the follow-up occasion, or whether this was related to a decrease in depressive symptoms and/or suicidality. Analysis of longterm variation of symptoms and biological factors in large numbers of psychiatric patients is highly warranted in the future.

Our study supports the hypothesis that a low-grade CNS inflammation induces production of QUIN, which has effects on glutamate neurotransmission and might lead to symptoms of suicidality. The positive correlation between the cytokine IL-6 and QUIN in suicide attempters is in line with findings in patients suffering from CNS infections, where both CSF IL-6 and QUIN are elevated (Heyes et al, 1995). Studies showing immunohistochemical evidence of microgliosis and an increased density of QUIN-immunoreactive microglial cells in suicide victims further support that brain inflammation, increased QUIN levels and suicide are linked (Steiner et al, 2008; Steiner et al, 2011). As an additional mechanism, reduced activity of the enzyme downstream of QUIN production, quinolinate phosphoribosyl-transferase, could potentially lead to an accumulation of QUIN. The enzyme degrades QUIN into nicotinic acid ribonucleotide and causes subsequent NAD + formation (Figure 1). It is also possible that peripheral inflammation may contribute to the synthesis of brain QUIN and KYNA. Plasma levels of kynurenine (precursor of QUIN and KYNA) are elevated in patients with major depressive disorder and a history of suicide attempts (Sublette et al, 2011). Kynurenine can pass the blood brain barrier and subsequently be metabolized into QUIN and KYNA by microglia and astrocytes. However, QUIN and KYNA themselves do not pass the blood-brain barrier and therefore CSF samples are needed to study the levels of these neuroactive metabolites in the CNS compartment 
(Schwarcz et al, 2012). An intriguing question is why QUIN is accumulating in excess over KYNA in suicide attempters, as both metabolites are generated in states of inflammation. In this respect, the regulation of enzymes in the kynurenine pathway deserves further investigation and could potentially depend on genetic variants (Claes et al, 2011). An imbalance in the activity of enzymes along the kynurenine pathway might explain why QUIN accumulates, or is produced in preference over KYNA in suicidal patients.

Considering that inflammation and dysregulation of glutamate neurotransmission might contribute to the pathophysiology of suicidal behavior, we propose that novel treatments of suicidality should be directed against these systems. Clinical trials using cyclo-oxygenase-2 inhibitors as add-on therapy in patients with treatmentresistant depression are promising (Akhondzadeh et al, 2009; Muller et al, 2006). Novel therapeutic strategies include inhibition of the enzyme IDO-1 or microglial activation (in order to reduce synthesis of QUIN), specific cytokine blockers or direct blockade of the NMDA-receptor (Haroon et al, 2012). These treatments could be tested in future trials enrolling patients based on suicidality. As mentioned above, the NMDA receptor antagonist ketamine was effective in initial trials treating suicidal symptoms. Moreover, the primary trigger of the elevated immune parameters in suicidality is currently unknown and once unraveled, it might constitute another drug target. In this context, it is of importance to keep in mind that suicidality is likely to be a complex phenomenon, not depending on a single cause. So-called distal risk factors include genetic loading, trait impulsivity and early traumatic life events, whereas proximal risk factors include psychological crisis or acute stress (Mann and Currier 2010); Gradus et al, 2010; Hawton and van Heerningen, 2009). It has been demonstrated that pro-inflammatory cytokines IL- 6 and IL-1 $\beta$ increase after acute stress (Steptoe et al, 2007). Psychological factors could thus potentially trigger a cascade of reactions ultimately leading to increased QUIN production. Another factor that recently has been associated with suicidality is the neurotrophic parasite T. gondii (Pedersen et al, 2012; Zhang et al, 2012). The parasite has been suggested to increase dopamine production as well as induce low-grade inflammation. These examples show that in order to achieve optimal symptom relief for suicidal patients, it would most likely be vital to identify and treat triggers of inflammation, as well as acute symptoms.

In summary, this is to our knowledge the first report of elevated QUIN, an endogenous NMDA agonist of microglial origin, in the CSF of suicide attempters. CSF QUIN was associated with the degree of suicidal intent and when a subgroup of patients came back for a follow-up visit, CSF QUIN levels had returned to normal. These findings suggest that increased CSF QUIN is specifically related to the pathophysiology of suicidality. As QUIN is an NMDA receptor agonist, our findings may provide a scientific rationale for the reduced suicidal ideation by the NMDAreceptor antagonist ketamine. Given that suicidal behavior is a major public health problem worldwide, with a poorly understood pathophysiology, our findings may lead to the development of novel tools to diagnose, follow and treat this source of morbidity and mortality across diagnostic boundaries.

\section{ACKNOWLEDGEMENTS}

This study was supported by the Swedish Research Council Grants No. 2009-4284 and 2011-4787 (Lena Brundin), 20025297 and 2008-2922 (Lil Träskman-Bendz), and 2009-7052 and 2011-4795 (Sophie Erhardt), the Province of Scania clinical state grants (ALF, for Lena Brundin and Lil Träskman-Bendz), the American Foundation for Suicide Prevention 2-DIG-00030-0608-1208 (Teodor Postolache), the Rebecca Cooper Foundation (Gilles Guillemin) and the National Health Medical Research Council in Australia (NHMRC) (Gilles Guillemin).

\section{DISCLOSURE}

The authors declare that, except for income received from the primary employers, no financial support or compensation has been received from any individual or corporate entity over the past three years for research or professional service; and there are no personal financial holdings that could be perceived as constituting a potential conflict of interest. None of the funding organizations listed above had any role in the design or conduct of the study, the data analysis, in the preparation of the manuscript or in the decision to submit the work for publication.

\section{REFERENCES}

Achim CL, Heyes MP, Wiley CA (1993). Quantitation of human immunodeficiency virus, immune activation factors, and quinolinic acid in AIDS brains. J Clin Invest 91: 2769-2775.

Akhondzadeh S, Jafari S, Raisi F, Nasehi AA, Ghoreishi A, Salehi B et al (2009). Clinical trial of adjunctive celecoxib treatment in patients with major depression: a double blind and placebo controlled trial. Depress Anxiety 26: 607-611.

American Psychiatric Association (1987). Diagnostic and Statistical Manual of Mental Disorders. American Psychiatric Press: Washington, DC.

Asberg M, Traskman L (1981). Studies of CSF 5-HIAA in depression and suicidal behaviour. Adv Exp Med Biol 133: 739-752.

Beck AT (1974). The Prediction of Suicide. Charles Press: Bowie, Md.

Beck AT, Davis JH, Frederick CJ, Perlin S, Pokorny AD, Schulman RE (1973). Classification and nomenclature. In: Resnik HLPHathorne BC (eds) Suicide Prevention in the Seventies. US Government Printing Office: Washington DC, pp 7-12.

Bremner JD, Narayan M, Anderson ER, Staib LH, Miller HL, Charney DS (2000). Hippocampal volume reduction in major depression. Am J Psychiatry 157: 115-118.

Brent DA, Emslie GJ, Clarke GN, Asarnow J, Spirito A, Ritz L et al (2009). Predictors of spontaneous and systematically assessed suicidal adverse events in the treatment of SSRI-resistant depression in adolescents (TORDIA) study. Am J Psychiatry 166: 418-426.

Bruffaerts R, Demyttenaere K, Hwang I, Chiu WT, Sampson N, Kessler RC et al (2011). Treatment of suicidal people around the world. Br J Psychiatry 199: 64-70.

Claes S, Myint AM, Domschke K, Del-Favero J, Entrich K, Engelborghs S et al (2011). The kynurenine pathway in major depression: haplotype analysis of three related functional candidate genes. Psychiatry Res 188: 355-360.

Da Cruz D, Pearson A, Saini P, Miles C, While D, Swinson N et al (2011). Emergency department contact prior to suicide in mental health patients. Emerg Med J 28: 467-471. 
de Carvalho LP, Bochet P, Rossier J (1996). The endogenous agonist quinolinic acid and the non endogenous homoquinolinic acid discriminate between NMDAR2 receptor subunits. Neurochem Int 28: 445-452.

Diazgranados N, Ibrahim L, Brutsche NE, Newberg A, Kronstein P, Khalife $S$ et al (2010a). A randomized add-on trial of an $\mathrm{N}$-methyl-D-aspartate antagonist in treatment-resistant bipolar depression. Arch Gen Psychiatry 67: 793-802.

Diazgranados N, Ibrahim LA, Brutsche NE, Ameli R, Henter ID, Luckenbaugh DA et al (2010b). Rapid resolution of suicidal ideation after a single infusion of an N-methyl-D-aspartate antagonist in patients with treatment-resistant major depressive disorder. J Clin Psychiatry 71: 1605-1611.

Duman RS, Aghajanian GK (2012). Synaptic dysfunction in depression: potential therapeutic targets. Science 338: 68-72.

Erhardt S, Olsson SK, Engberg G (2009). Pharmacological manipulation of kynurenic acid: potential in the treatment of psychiatric disorders. CNS Drugs 23: 91-101.

Ernst C, Mechawar N, Turecki G (2009). Suicide neurobiology. Prog Neurobiol 89: 315-333.

Fragoso YD, Frota ER, Lopes JS, Noal JS, Giacomo MC, Gomes S et al (2010). Severe depression, suicide attempts, and ideation during the use of interferon beta by patients with multiple sclerosis. Clin Neuropharmacol 33: 312-316.

Freedenthal S (2008). Assessing the wish to die: a 30-year review of the suicide intent scale. Arch Suicide Res 12: 277-298.

Gradus JL, Qin P, Lincoln AK, Miller M, Lawler E, Sorensen HT et al (2010). Acute stress reaction and completed suicide. Int J Epidemiol 39: 1478-1484.

Guillemin GJ (2012). Quinolinic acid, the inescapable neurotoxin. FEBS J 279: 1356-1365.

Haroon E, Raison CL, Miller AH (2012). Psychoneuroimmunology meets neuropsychopharmacology: translational implications of the impact of inflammation on behavior. Neuropsychopharmacology 37: 137-162.

Hashimoto K (2009). Emerging role of glutamate in the pathophysiology of major depressive disorder. Brain Res Rev 61: 105-123.

Hawton K, van Heeringen K (2009). Suicide. Lancet 373: 1372-1381.

Heyes MP, Saito K, Crowley JS, Davis LE, Demitrack MA, Der M et al (1992). Quinolinic acid and kynurenine pathway metabolism in inflammatory and non-inflammatory neurological disease. Brain 115(Pt 5): 1249-1273.

Heyes MP, Saito K, Milstien S, Schiff SJ (1995). Quinolinic acid in tumors, hemorrhage and bacterial infections of the central nervous system in children. J Neurol Sci 133: 112-118.

Hilmas C, Pereira EF, Alkondon M, Rassoulpour A, Schwarcz R, Albuquerque EX (2001). The brain metabolite kynurenic acid inhibits alpha7 nicotinic receptor activity and increases nonalpha7 nicotinic receptor expression: physiopathological implications. J Neurosci 21: 7463-7473.

Ibrahim L, Diazgranados N, Franco-Chaves J, Brutsche N, Henter ID, Kronstein P et al (2012). Course of improvement in depressive symptoms to a single intravenous infusion of ketamine vs add-on riluzole: results from a 4-week, double-blind, placebocontrolled study. Neuropsychopharmacology 37: 1526-1533.

Janelidze S, Mattei D, Westrin A, Traskman-Bendz L, Brundin L (2011). Cytokine levels in the blood may distinguish suicide attempters from depressed patients. Brain Behav Immun 25: 335-339.

Larkin GL, Beautrais AL (2011). A preliminary naturalistic study of low-dose ketamine for depression and suicide ideation in the emergency department. Int $J$ Neuropsychopharmacol 14: $1127-1131$.

Lindqvist D, Janelidze S, Hagell P, Erhardt S, Samuelsson M, Minthon L et al (2009). Interleukin-6 is elevated in the cerebrospinal fluid of suicide attempters and related to symptom severity. Biol Psychiatry 66: 287-292.
Mann JJ, Currier DM (2010). Stress, genetics and epigenetic effects on the neurobiology of suicidal behavior and depression. Eur Psychiatry 25: 268-271.

McKinnon MC, Yucel K, Nazarov A, MacQueen GM (2009). A meta-analysis examining clinical predictors of hippocampal volume in patients with major depressive disorder. J Psychiatry Neurosci 34: 41-54.

Montgomery SA, Asberg M (1979). A new depression scale designed to be sensitive to change. Br J Psychiatry 134: 382-389.

Muller N, Schwarz MJ, Dehning S, Douhe A, Cerovecki A, Goldstein-Muller B et al (2006). The cyclooxygenase-2 inhibitor celecoxib has therapeutic effects in major depression: results of a double-blind, randomized, placebo controlled, add-on pilot study to reboxetine. Mol Psychiatry 11: 680-684.

Murrough JW, Perez AM, Pillemer S, Stern J, Parides MK, Aan Het Rot $M$ et al (2012). Rapid and longer-term antidepressant effects of repeated ketamine infusions in treatmentresistant major depression. Biol Psychiatry; e-pub ahead of print 26 July 2012. doi: 10.1016/j.biopsych.2012.06.02.

Nock MK, Borges G, Bromet EJ, Cha CB, Kessler RC, Lee S (2008). Suicide and suicidal behavior. Epidemiol Rev 30: 133-154.

Olsson SK, Samuelsson M, Saetre P, Lindstrom L, Jonsson EG, Nordin C et al (2010). Elevated levels of kynurenic acid in the cerebrospinal fluid of patients with bipolar disorder. J Psychiatry Neurosci 35: 195-199.

Oxenkrug GF (2010). Tryptophan kynurenine metabolism as a common mediator of genetic and environmental impacts in major depressive disorder: the serotonin hypothesis revisited 40 years later. Isr J Psychiatry Relat Sci 47: 56-63.

Pedersen MG, Mortensen PB, Norgaard-Pedersen B, Postolache TT (2012). Toxoplasma gondii infection and self-directed violence in mothers. Arch Gen Psychiatry 69: 1123-1130.

Price RB, Nock MK, Charney DS, Mathew SJ (2009). Effects of intravenous ketamine on explicit and implicit measures of suicidality in treatment-resistant depression. Biol Psychiatry 66: 522-526.

Raison CL, Borisov AS, Majer M, Drake DF, Pagnoni G, Woolwine BJ et al (2009). Activation of central nervous system inflammatory pathways by interferon-alpha: relationship to monoamines and depression. Biol Psychiatry 65: 296-303.

Raison CL, Dantzer R, Kelley KW, Lawson MA, Woolwine BJ, Vogt G et al (2010). CSF concentrations of brain tryptophan and kynurenines during immune stimulation with IFN-alpha: relationship to CNS immune responses and depression. Mol Psychiatry 15: 393-403.

Sanacora G, Zarate CA, Krystal JH, Manji HK (2008). Targeting the glutamatergic system to develop novel, improved therapeutics for mood disorders. Nat Rev Drug Discov 7: 426-437.

Schwarcz R, Bruno JP, Muchowski PJ, Wu HQ (2012). Kynurenines in the mammalian brain: when physiology meets pathology. Nat Rev Neurosci 13: 465-477.

Sher L (2011). Brain-derived neurotrophic factor and suicidal behavior. QJM 104: 455-458.

Smythe GA, Braga O, Brew BJ, Grant RS, Guillemin GJ, Kerr SJ et al (2002). Concurrent quantification of quinolinic, picolinic, and nicotinic acids using electron-capture negative-ion gas chromatography-mass spectrometry. Anal Biochem 301: 21-26.

Stefansson J, Nordstrom P, Jokinen J (2012). Suicide Intent Scale in the prediction of suicide. J Affect Disord 136: 167-171.

Steiner J, Bielau H, Brisch R, Danos P, Ullrich O, Mawrin C et al (2008). Immunological aspects in the neurobiology of suicide: elevated microglial density in schizophrenia and depression is associated with suicide. J Psychiatr Res 42: 151-157.

Steiner J, Walter M, Gos T, Guillemin GJ, Bernstein HG, Sarnyai Z et al (2011). Severe depression is associated with increased microglial quinolinic acid in subregions of the anterior cingulate gyrus: evidence for an immune-modulated glutamatergic neurotransmission? J Neuroinflammation 8: 94. 
Steptoe A, Hamer M, Chida Y (2007). The effects of acute psychological stress on circulating inflammatory factors in humans: a review and meta-analysis. Brain Behav Immun 21: 901-912.

Stone TW (1993). Neuropharmacology of quinolinic and kynurenic acids. Pharmacol Rev 45: 309-379.

Sublette ME, Galfalvy HC, Fuchs D, Lapidus M, Grunebaum MF, Oquendo MA et al (2011). Plasma kynurenine levels are elevated in suicide attempters with major depressive disorder. Brain Behav Immun 25: 1272-1278.

Traskman L, Asberg M, Bertilsson L, Sjostrand L (1981). Monoamine metabolites in CSF and suicidal behavior. Arch Gen Psychiatry 38: 631-636.

Wichers MC, Koek GH, Robaeys G, Verkerk R, Scharpe S, Maes M (2005). IDO and interferon-alpha-induced depressive symptoms: a shift in hypothesis from tryptophan depletion to neurotoxicity. Mol Psychiatry 10: 538-544.
Zarate Jr CA, Brutsche NE, Ibrahim L, Franco-Chaves J, Diazgranados N, Cravchik A et al (2012). Replication of ketamine's antidepressant efficacy in bipolar depression: a randomized controlled add-on trial. Biol Psychiatry 71: 939-946.

Zhang Y, Traskman-Bendz L, Janelidze S, Langenberg P, Saleh A, Constantine $\mathrm{N}$ et al (2012). Toxoplasma gondii immunoglobulin $\mathrm{G}$ antibodies and nonfatal suicidal self-directed violence. J Clin Psychiatry 73: 1069-1076.

(c) (1)(8) $\Theta$ This work is licensed under the Creative Commons Attribution-NonCommercial-No Derivative Works 3.0 Unported License. To view a copy of this license, visit http:// creativecommons.org/licenses/by-nc-nd/3.0/

Supplementary Information accompanies the paper on the Neuropsychopharmacology website (http://www.nature.com/npp) 